\title{
Lipid content and fatty acid profiles in ten species of microalgae
}

\author{
Contenido de lípidos y perfil de ácidos grasos en diez especies de microalgas \\ Silvana Ohse ${ }^{1 *}$, Roberto Bianchini Derner ${ }^{2}$, Renata Ávila Ozório ${ }^{2}$, Rafaela Gordo Corrêa ${ }^{3}$, \\ Eliana Badiale Furlong ${ }^{4}$, Paulo Cesar Roberto Cunha ${ }^{5}$
}

\begin{abstract}
The present study aimed to evaluate the lipid content and fatty acid profiles of 9 marine species (Nannochloropsis oculata, Thalassiosira pseudonana, Phaeodactylum tricornutum, Isochrysis galbana, Tetraselmis suecica, Tetraselmis chuii, Chaetoceros muelleri, Thalassiosira fluviatilis and Isochrysis sp.) and 1 freshwater microalga species (Chlorella vulgaris) that were cultivated in Erlenmeyer flasks with $800 \mathrm{~mL}$ of culture medium under stationary autotrophic conditions while exposed to a continuous photon flux density of approximately $175 \mu \mathrm{mol} \mathrm{m} \mathrm{m}^{-2} \mathrm{~s}^{-1}$, a temperature of $25 \pm 2{ }^{\circ} \mathrm{C}$ and constant airflow. $N$. oculata and T. pseudonana exhibited the highest lipid content, although they had a high proportion of monounsaturated fatty acids relative to polyunsaturated fatty acids. C. vulgaris and Isochrysis sp. exhibited high levels of polyunsaturated fatty acids, whereas P. tricornutum, N. oculata and $T$. suecica exhibited the highest eicosapentaenoic acid levels, and I. galbana and C. muelleri had the highest docosahexaenoic fatty acid levels. The freshwater species $C$. vulgaris had the highest levels of linoleic and linolenic acids, followed by the marine species T. chuii.
\end{abstract}

Key words: lipids, fatty acid profile, microalgae.

\section{RESUMEN}

El presente estudio pretende como objetivo evaluar el perfil de ácidos grasos y contenido de lípidos de nueve microalgas marinas (Nannochloropsis oculata, Thalassiosira pseudonana, Phaeodactylum tricornutum, Isochrysis galbana, Tetraselmis suecica, Tetraselmis chuii, Chaetoceros muelleri, Thalassiosira fluviatilis $e$ Isochrysis sp.) y una de agua dulce (Chlorella vulgaris) y la cultura autotrófica estacionaria, usando frascos que contienen $800 \mathrm{ml}$ de medio de cultivo, proporcionando constante la densidad del flujo de fotones de iluminación en los cultivos de alrededor de $175 \mathrm{~mol} \mathrm{~m} \mathrm{~m}^{-2} \mathrm{~s}^{-1}$, temperatura $25 \pm 2{ }^{\circ} \mathrm{C}$ y aireación. La especie N. oculata y T. pseudonana mostraron los niveles más altos de lípidos, pero con una alta proporción de ácidos grasos monoinsaturados en relación con poliinsaturados. La especie C. vulgaris e Isochrysis sp. mostraron altos niveles de ácidos grasos poliinsaturados; P. tricornutum, N. oculata y T. suecica revelaron los niveles más altos de ácido eicosapentaenoico y C. muelleri e I. galbana alcanzaron los más altos niveles del ácido graso docosahexaenoico. C. vulgaris reveló un mayor contenido de ácidos linoleico y linolénico, seguido de las especies marinas T. chuii.

Palabras clave: lipídicos, perfil de ácidos grasos, microalga.

\section{Introduction}

Microalgae are important constituents of ecosystems that range from marine and freshwater to desert and from hot to cold, and thus possess varied compositions of several chemical components such as proteins, lipids, fatty acids, carbohydrates and pigments. The concentrations of each component depend on the nature of the organism, the environmental or culture conditions and the physiological state of the culture itself. Differences in the chemical composition of microalgae cultures, including lipid content and fatty acid profiles, can be found not only between different species but also within the same species; these differences depend on the composition of the culture medium,

\footnotetext{
Departamento de Fitotecnia e Fitossanidade, Universidade Estadual de Ponta Grossa, Ponta Grossa/PR, Brasil.

2 Universidade Federal de Santa Catarina, Centro de Ciências Agrárias, Departamento de Aqüicultura, Laboratório de Camarões Marinhos, Florianópolis/SC, Brasil.

3 Universidade Federal de Santa Catarina. Florianópolis/SC, Brasil.

4 Universidade Federal de Rio Grande, Rio Grande/RS, Brasil.

5 CENPES-PETROBRAS, Ilha do Fundão, Rio de Janeiro/RJ. Brasil.

* Corresponding Author: sohse@ uepg.br*
}

Fecha de Recepción: 6 Agosto, 2013.

Fecha de Aceptación: 27 Noviembre, 2014. 
airflow, carbon content, luminosity, photoperiod, culture temperature and $\mathrm{pH}$, among others (Miao $\& \mathrm{Wu}, 2004)$.

Some cyanobacteria produce large amounts of polyunsaturated fatty acids (PUFAs; 20-60\%). Saturated and monounsaturated fatty acids (SFAs and MUFAs, respectively) are among the most prominent stored lipid components in eukaryotic algae and generally reach values greater than $80 \%$ of the total lipid fraction (Richmond, 2004). Some marine microalgae species contain high levels of long-chain polyunsaturated fatty acids (LC-PUFAs) in their lipid fractions, such as eicosapentaenoic (EPA - 20:5 ${ }_{\mathrm{n}-3}$ ) and docosahexaenoic acids (DHA $-22: 6_{\mathrm{n}-3}$ ) (Medina et al., 1998).

Studies have shown that the ingestion of algae-derived $\omega$-3 fatty acids has beneficial effects on visual and neural development and on the prevention of diseases such as heart conditions, hypertension, cancer, diabetes, cystic fibrosis, asthma, arthritis, depression and schizophrenia, a finding that is also reflected by the large number of microalgae-based products that have biological activities in humans (Derner et al., 2006). Ethanol or supercritical fluid lipid extraction has increased in commercial importance with regard to the production of lipid-based cosmetic formulations such as creams or lotions, because these provide both nutritional and protective effects to the skin. Other microalgae-derived lipids, including glycolipids and phospholipids, should not be overlooked in future skin care developments.

Many microalgae species are used in food production because they produce several substances, including vitamins, mineral salts, pigments, lipids and fatty acids. The main applications of microalgaderived fatty acids include the enrichment of fish food (e.g., in aquiculture), possible uses in biodiesel production, an essential fatty acid source in the human diet as both a food additive and a human health supplement, biological carbon sequestration and sewage treatment. The amounts of commercially interesting compounds that can be obtained from microalgae are unpredictable, thus making studies of species and chemical composition of microalgae indispensable, in addition to a strong interest in clean, sustainable and organic technologies to generate products for human use (Derner et al., 2006).

Much is known about the uses of certain fatty acids, especially long-chained ones. Still, few studies on the lipid contents and fatty acid profiles of microalgae have been performed in Brazil. Thus the present study aims to identify differences between microalgae species with respect to the above characteristics.

\section{Materials and Methods}

The experiments were performed in a cell culture room at the Department of Food Science (Departamento de Ciência dos Alimentos-CAL), Federal University of Santa Catarina (Universidade Federal de Santa Catarina-UFSC) in Florianópolis, Santa Catarina State (SC), Brazil. The studied microalgae included the freshwater species Chlorella vulgaris and the marine species Nannochloropsis oculata, Thalassiosira pseudonana, Phaeodactylum tricornutum, Isochrysis galbana, Tetraselmis suecica, Tetraselmis chuii, Chaetoceros muelleri, Thalassiosira fluviatilis and Isochrysis sp. The experimental design comprised randomised blocks of time with 3 repetitions.

The culture room was maintained at a temperature of $25 \pm 2{ }^{\circ} \mathrm{C}$ with continuous artificial culture radiation $\left(75 \mu \mathrm{mol} \mathrm{m} \mathrm{m}^{-2} \mathrm{~s}^{-1}\right.$ on the first day of culture and increasing to $150 \mu \mathrm{mol} \mathrm{m} \mathrm{m} \mathrm{s}^{-1}$ from the second day until harvest). Modified WC medium was used to culture C. vulgaris (Guillard \& Lorenzen, 1972), and $\mathrm{f} / 2$ Guillard's medium was used to culture the marine species (Guillard, 1975).

The experimental phase included the inoculation of a predetermined volume of algal culture, depending on the desired initial density, into $800 \mathrm{~mL}$ of culture medium. For each repetition, this $800 \mathrm{~mL}$ volume was added to $1000 \mathrm{~mL}$ Erlenmeyer flasks, autoclaved for $30 \mathrm{~min}$ at $121^{\circ} \mathrm{C}$ and stored for $48 \mathrm{~h}$ at $25^{\circ} \mathrm{C}$. The freshwater culture medium was corrected to $\mathrm{pH}$ 6.5 with $10 \% \mathrm{HCl}$; the saltwater medium remained at approximately $\mathrm{pH} 8.0$, thus no correction was necessary. Culture airflow was provided with a mini compressor (Boyu Acq-003 50/L).

To harvest the cultures, the culture in each flask was centrifuged twice for $20 \mathrm{~min}$ at $4000 \mathrm{rpm}$ and $10{ }^{\circ} \mathrm{C}$. The samples were frozen, lyophilised, centrifuged, dried in an incubator at $50{ }^{\circ} \mathrm{C}$ and ground, followed by lipid extraction. Fatty acid methyl esters were obtained by lipid esterification as previously described by Metcalfe \& Schimitz (1966), and the fatty acid profiles were determined by gas chromatography (Varian $3400 \mathrm{CX}$, equipped with a flame ionisation detector; Varian Medical Systems, Inc., Palo Alto, CA, USA). 
The lipid content and fatty acid profile data obtained were submitted to a variance analysis and Tukey's test to identify differences between the mean values of each treatment. The ESTAT software package was used for this purpose.

\section{Results and Discussion}

The total lipid content per dry mass (TLDM) levels were found to be higher in N. oculata than in T. suecica, T. fluviatilis, C. muelleri, T. chuii and C. vulgaris (Table 1). Servel et al. (1994) reported higher levels in I. galbana (25.6\%) and C. calcitrans $(11.8 \%)$ and lower levels in Nannochloropsis sp. (5.6\%). Mata et al. (2010) reported TLDM values of $22.7-29.7 \%$ in N. oculata, $18-57 \%$ in P. tricornutum, $20.6 \%$ in T. pseudonana, $7 \%$ to $40 \%$ in Isochrysis galbana, $8.5-23 \%$ in Tetraselmis suecica, 5-58\% in Chlorella vulgaris, from 7.1-33\% in Isochrysis sp. and $33.6 \%$ for C. muelleri, thus corroborating the data obtained in the present study, in which only C. muelleri exhibited a lower value (Table 1).

According to Michael and Borowitzka (1988), the ideal TLDMs for the different classes of algae cited are as follows: Cyanophyceae 2-23\%, Chrysophyceae 12-72\%, Prymnesiophyceae 5-48\%, Cryptophyceae 3-17\%, Xanthophyceae 6-16\%, Bacillariophyceae 1-39\%, Chlorophyceae 1-70\% and Euglenophyceae 1-17\%. In the present study, the highest TLDM among the Bacillariophyceae was found in T. pseudonana $(24.67 \%)$, among Prymnesiophyceae in Isochrysis sp. (21.08\%) and among Chlorophyceae in C. vulgaris (16.08\%), which agrees with the wide range of values cited above (Table 1).

Renaud et al. (1999) studied 18 microalgae species and found TLDM values of $17 \%$ in Chaetoceros sp. with a mean percentage of $15.4 \%$ in the order Bacillariophyceae, $13.2 \%$ in Tetraselmis sp. with a mean percentage of $12.3 \%$ in Prasinophyceae, $23.4 \%$ in Isochrysis sp. and $17.5 \%$ in Prymnesiophyceae. In the present study, a similar value was obtained for Isochrysis sp. with a mean percentage of $20.3 \%$, and a lower value for C. muelleri, despite a very similar overall average percentage in the order Bacillariophyceae (15.6\%), as well as a lower value for the order Prymnesiophyceae (T. suecica and T. Chuii) with a mean percentage of $9.6 \%$ (Table 1). Renaud et al. (2002) obtained TLDMs of $20.7 \%$ and $16.8 \%$ for Isochrysis sp. and Chaetoceros sp., respectively, which are similar and higher, respectively, than the values obtained for the same genera in the present study (Table 1). Servel et al. (1994) reported a lower TLDM in T. Suecica, and Castro Araújo \& Garcia (2005) found higher content in Chaetoceros sp.

Table 1. Total lipid content as percentage of dry mass (TLDM), saturated fatty acid content as percentage of total lipids (SFAC/TL) and total fatty acids (SFAC/TFA) and monounsaturated and polyunsaturated fatty acid content as percentages of total fatty acids (MUFAC/TFA and PUFAC/TFA, respectively) and of total lipids

(MUFAC/TL and PUFAC/TL, respectively) in ten microalgae species.

\begin{tabular}{|c|c|c|c|c|c|c|c|}
\hline Species & TLDM & $\begin{array}{c}\text { SFAC/ } \\
\text { TL }\end{array}$ & $\begin{array}{c}\text { SFAC/ } \\
\text { TFA }\end{array}$ & $\begin{array}{c}\text { MUFAC/ } \\
\text { TFA }\end{array}$ & MUFAC/TL & $\begin{array}{c}\text { PUFAC/ } \\
\text { TFA }\end{array}$ & $\begin{array}{c}\text { PUFAC/ } \\
\text { TL }\end{array}$ \\
\hline & \multicolumn{7}{|c|}{$(\%)$} \\
\hline T. pseudonana & $24.7 \mathrm{ab}$ & $9.0 \mathrm{ab}$ & $36.7 \mathrm{~cd}$ & $44.7 \mathrm{a}$ & $11.0 \mathrm{a}$ & $12.9 \mathrm{~cd}$ & $3.2 \mathrm{bc}$ \\
\hline N. oculata & $27.2 \mathrm{a}$ & $12.7 \mathrm{a}$ & $46.9 \mathrm{abc}$ & $44.5 \mathrm{a}$ & $12.1 \mathrm{a}$ & $8.8 \mathrm{~d}$ & $2.4 \mathrm{bcd}$ \\
\hline C. muelleri & $10.2 \mathrm{~cd}$ & $3.0 \mathrm{c}$ & $29.4 \mathrm{~d}$ & $36.3 \mathrm{ab}$ & $3.7 \mathrm{bcd}$ & $15.0 \mathrm{bcd}$ & $1.5 \mathrm{~cd}$ \\
\hline T. suecica & $8.4 \mathrm{~d}$ & $3.5 \mathrm{c}$ & $41.9 \mathrm{bc}$ & $30.8 \mathrm{~b}$ & $2.6 \mathrm{~cd}$ & $26.0 \mathrm{abc}$ & $2.2 \mathrm{bcd}$ \\
\hline I. galbana & $19.5 \mathrm{abc}$ & $4.6 \mathrm{a}$ & $58.0 \mathrm{a}$ & $28.8 \mathrm{~b}$ & $5.6 \mathrm{~b}$ & $12.5 \mathrm{~cd}$ & $2.5 \mathrm{bcd}$ \\
\hline Isochrysis sp. & $21.1 \mathrm{ab}$ & $8.8 \mathrm{ab}$ & $41.7 \mathrm{bc}$ & $24.4 \mathrm{~b}$ & $5.2 \mathrm{bc}$ & $27.3 \mathrm{ab}$ & $5.8 \mathrm{a}$ \\
\hline T. chuii & $10.7 \mathrm{~cd}$ & $5.4 \mathrm{bc}$ & $50.0 \mathrm{ab}$ & $24.2 \mathrm{~b}$ & $2.6 \mathrm{~cd}$ & $25.6 \mathrm{abc}$ & $2.7 \mathrm{bcd}$ \\
\hline C. vulgaris & $16.1 \mathrm{bcd}$ & $6.5 \mathrm{bc}$ & $40.2 \mathrm{bcd}$ & $23.5 \mathrm{~b}$ & $3.8 \mathrm{bc}$ & $31.9 \mathrm{a}$ & $5.1 \mathrm{a}$ \\
\hline T. fluviatilis & $9.3 \mathrm{~d}$ & $5.3 \mathrm{bc}$ & $57.3 \mathrm{a}$ & $23.2 \mathrm{~b}$ & $2.2 \mathrm{~cd}$ & $15.0 \mathrm{bcd}$ & $1.4 \mathrm{~d}$ \\
\hline P. tricornutum & $18.1 \mathrm{abcd}$ & $8.5 \mathrm{ab}$ & $46.8 \mathrm{abc}$ & $4.2 \mathrm{c}$ & $0.8 \mathrm{~d}$ & $18.3 \mathrm{abcd}$ & $3.3 \mathrm{~b}$ \\
\hline Mean & 16.52 & 7.4 & 44.9 & 28.46 & 4.95 & 19.3 & 3.0 \\
\hline $\mathrm{CV}(\%)$ & 20.30 & 22.41 & 9.16 & 15.78 & 20.45 & 25.1 & 19.71 \\
\hline
\end{tabular}

* Mean values not followed by the same letter differ from each other according to Tukey's test, with an error probability of 5\%. 
Higher TLDM values for the genus Isochrysis were reported by Valenzuela-Espinoza et al. (2002) and Servel et al. (1994). Some studies also reported higher TLDM values in the genus Chaetoceros than those obtained in the present study (Saavedra \& Voltolina, 1994; Servel et al., 1994), whereas others reported similar values (López-Elías et al., 2003; López-Elías et al., 2005). Rebolloso-Fuentes et al. (2001) reported TLDM values that were similar to those obtained in the present study for P. tricornutum (Table 1).

The species $C$. vulgaris was found to have TLDM values compatible with some studies (Richmond, 2004) and lower than those reported by other research groups (Medina et al., 1998). According to Richmond (2004), the variations in TLDM values are due to differences in culture conditions, cultivation type, nutrient concentration, growth rate, life cycle phase, environmental conditions and the state of the cells in the culture.

The saturated fatty acid content of the total lipids (SFAC/TL) and the total fatty acids (SFAC/TFA) were calculated and are expressed as $\%(\mathrm{p} / \mathrm{p}) . N$. oculata exhibited the highest SFAC/TL, although this value was not significantly different from those of the species T. pseudonana, Isochrysis sp., I. galbana and P. tricornutum. I. galbana exhibited the highest SFAC/TFA, although this value did not differ significantly from those of the species $T$. fluviatilis, $P$. tricornutum, $N$. oculata and T. chuii (Table 1). The species I. galbana and T. fluviatilis contain more than 50\% SFA, including both TL and TFA. In other words, they produce lower levels of unsaturated fatty acids (UFA), which are the most desirable for human and animal nutrition. LópezElías et al. (2003) found a 60\% SFAC/TL level in Chaetoceros sp., a higher value than that observed in the present study (Table 1).

The SFAC/TFA and SFAC/TL values listed in Table 1 show that $I$. galbana exhibited the highest SFAC/TFA, although it did not differ significantly from $T$. fluviatilis, T. chuii, N. oculata or $P$. tricornutum. N. oculata exhibited higher SFAC/TL, but again did not differ significantly from I. galbana, T. pseudonana, Isochrysis sp. or P. tricornutum. According to Qi et al. (2002) I. galbana is rich in PUFAs, however this finding did not agree with the present study, because the highest content observed was that of SFAs, with an average of $58 \%$ of the total fatty acids (Table 1). Nevejan et al. (2003) found a lower SFA content in I. galbana $(33.7 \%$ of total fatty acids).

Regarding the MUFA content found in the total lipids (MUFAC/TL) and the total fatty acids (MUFAC/TFA), the species $N$. oculata exhibited a higher MUFAC/TL that did not differ significantly from that of T. pseudonana, and T. pseudonana exhibited the highest MUFAC/TFA, although this was not significantly different from those of the species N. oculata and C. muelleri (Table 1). LópezElías et al. (2003) determined a mean MUFAC/ $\mathrm{TL}$ of $5.23 \%$ for Chaetoceros sp., which is lower than the value obtained in the present study for the same genus (Table 1). Renaud et al. (1999) reported MUFAC/TFA values of $23.3 \%, 14.7 \%$ and $11.3 \%$ in Chaetoceros sp., Tetraselmis sp. and Isochrysis sp., respectively; these are lower values than those obtained in the present study for the same genera (Table 1), whereas Renaud et al. (2002) reported similar values for Chaetoceros sp. (39.4\%) and Isochrysis sp. (20.5\%).

The MUFAC/TFA of I. galbana, T. suecica, Nannochloropsis sp. and C. calcitrans were determined by Servel et al. (1994) to be $14.8 \%$, $17.1 \%, 24.0 \%$ and $29.1 \%$, respectively, which are lower than, respectively, those obtained in the present study for the same genera (Table 1). Qi et al. (2002) and Nevejan et al. (2003) obtained MUFAC/TFAs of $20.7 \%$ and $23.2 \%$, respectively, for I. galbana, which are very similar to the values obtained in the present study for that species (Table 1). Evaluating the MUFAC/TFA in N. oculata, P. tricornutum and T. pseudonana, Tonon et al. (2002) obtained values of $47.26 \%, 34.61 \%$ and $31.45 \%$, respectively, which are similar to, lower than and higher than, respectively, those obtained for the same species in the present study (Table 1).

Regarding the PUFA content found in total fatty acids (PUFAC/TFA) and total lipids (PUFAC/TL), the species $C$. vulgaris exhibited a higher PUFAC/ TFA that was not significantly different from those of the species Isochrysis sp., T. suecica, T. chuii and P. Tricornutum. Isochrysis sp. exhibited a higher PUFAC/TL that did not differ significantly from that of $C$. vulgaris. The PUFAC/TFA determined for $C$. vulgaris was similar to that reported by Moraes \& Vieira Costa (2008). N. oculata exhibited a lower PUFAC/TFA; however, because it accumulated higher TLDM in our study, the PUFAC/TL value did not match the earlier reports (Table 1). 
Renaud et al. (1999) obtained PUFAC/TFA values of $47.2 \%, 56.35 \%$ and $56.4 \%$ in Chaetoceros sp., Tetraselmis sp. and Isochrysis sp., respectively, which were higher than the values obtained in the present study for the same genera (Table 1). Further, Renaud et al. (2002) reported PUFAC/ TFAs of $19.5 \%$ and $37.4 \%$ in Chaetoceros sp. and Isochrysis sp., respectively, and López-Elías et al. (2003) reported a PUFAC/TFA value of $34.77 \%$ in Chaetoceros sp.; these values are also higher than those obtained in the present study (Table 1). Saavedra \& Voltolina (1994) found MUFAC/TFA, SFAC/TFA and PUFAC/TFA values of $47.83 \%$, $43.84 \%$ and $0.93 \%$, respectively, in Chaetoceros sp., a trend that was also observed in the present study, although lower values for MUFAC/TFA $(36.3 \%)$ and SFAC/TFA $(29.35 \%)$ and higher values for PUFAC/TFA (15.01\%) were also observed (Table 1).

Servel et al. (1994) obtained PUFAC/TFA values of $61.5 \%, 58.6 \%, 53.3 \%$ and $32.5 \%$ for I. galbana, T. suecica, Nannochloropsis sp. and C. calcitrans, respectively; these comprised $17.0 \%, 20.9 \%, 2.8 \%$ and $8.7 \%$ of the total lipids. The values reported by the authors are higher than those listed in Table 1, in which the only similar value was that obtained for the PUFAC/TL of $N$. oculata. According to Qi et al. (2002), I. galbana is rich in PUFAs with a total value of $48.9 \%$, which is higher than the average obtained for this species in the present study (Table 1). Nevejan et al. (2003) also observed high PUFAC/ TFA values for I. galbana (41.7\%). Tonon et al. (2002) obtained PUFAC/TFA values of $12.13 \%$, $33.08 \%$ and $21.20 \%$ for $N$. oculata, P. tricornutum and T. pseudonana, respectively; however, the values obtained in the present study were lower for the same species (Table 1). The highest fatty acid content was the SFA 18:0 stearic acid value obtained for T. fluviatilis, $37.95 \%$, which was followed by the MUFA $16: 1$ of $16.12 \%$ (Table 2).

C. muelleri is a prominent food source for animals of high commercial value, especially crustaceans and bivalve molluscs. The importance of this species is due to its fatty acid profile, which comprises $5-20 \% 20: 5_{(\mathrm{n}-3)}, 0.2-1 \% 22: 6_{(\mathrm{n}-3)}$ and less than $0.2 \% 22: 4_{(\mathrm{n}-6)}$ (Brown et al., 1997). This profile is very different from that found in the present study for the same species, which showed higher content of the fatty acids 16:1 (36.33\%), 18:0 (14.38\%), 14:0 (11.87\%) and the PUFA 20:3 (9.69\%) (Table 2). Servel et al. (1994) observed a high 16:1 palmitoleic acid content in this genus, however that study also found high levels of the PUFA 20:5 ${ }_{n-3}$ /EPA (34\%). Saavedra \& Voltolina (1994) found a higher percentage of the fatty acid $16: 1(47.83 \%)$, followed by $16: 0(21.82 \%)$ and $14: 0$ (18.29\%) in Chaetoceros sp. The highest percentage found for this species in the present study was that of the fatty acid 16:1 (36.33\%), this was followed by $18: 0(14.38 \%), 14: 0(11.87 \%)$ and a considerable percentage of the PUFA 20:3 (9.69\%).

Rousch et al. (2003) compared P. tricornutum and $C$. muelleri and observed a higher number of fatty acids in the $C$. muelleri profile, in which 14:0, 16:0, 16:1, 16:2, 16:3, 18:0,18:1 ${ }_{(\mathrm{n}-9)}, 18: 2_{(\mathrm{n}-}$ ${ }_{6)}$ and $20: 5_{(\mathrm{n}-3)}$ were the main fatty acids detected. López-Elías et al. (2003) obtained the following average fatty acid percentages for Chaetoceros sp. (in decreasing order): 14:0 (31.57\%), 16:0 (20.46\%), 18:3 (16.58\%), 20:5 (10.22\%), 18:0 (7.98\%), 18:1 (5.23\%), 20:4 (4.14\%), 18:2 (1.73\%), $22: 6(1.56 \%)$ and $18: 4(0.53 \%)$. This profile differs strongly from the one found in the present study, especially because no 16:1/palmitoleic acid was detected (Table 2). Renaud et al. (1999) obtained a higher SFAC value for 14:0 (18.8\%), MUFAC for $18: 1_{\mathrm{n}-7}(20.2 \%)$ and PUFAC for $20: 5_{\mathrm{n}-3}(16.7 \%)$ in Chaetoceros sp. Similarly, Renaud et al. (2002) observed a higher SFAC value for 14:0 (23.6\%), MUFAC for $16: 1_{n-7}(36.5 \%)$ and PUFAC for $20: 5_{n-3}$ (8.0\%) in cultivated Chaetoceros sp. There were similarities with respect to MUFAC; specifically, in addition to a SFAC value of $14.28 \%$ for $16: 0$, 18:0 was also predominant with a value of $14.25 \%$. The predominant PUFAC was 20:3, with $10.0 \%$ of the total fatty acids.

In the present study the mean percentages of SFAs (46.88\%) and MUFAs (44.49\%) found in $N$. oculata were similar, however, the PUFA percentage $(8.76 \%)$ was much lower (Table 1). 16:0 palmitic acid was the most abundant SFA found in $N$. oculata, comprising $33.17 \%$ of the total fatty acids. The MUFA 16:1 palmitoleic acid was the second most abundant, comprising $30.96 \%$ of the total fatty acid content, followed by $18: 1$ with $13.53 \%$. The long chain PUFA (LC-PUFA) present in the highest amounts was 20:5 $\mathrm{n}-3$ /EPA (eicosapentanoic), which represented $5.36 \%$ of the total fatty acid content (Table 2). Similar results were reported by Servel et al. (1994) for Nannochloropsis sp., however the EPA content in that study was much higher $(30.1 \%)$ than the value obtained in the present study. 
Table 2. Fatty acid profiles ( $\%$ of total fatty acids) obtained from ten microalgae species.

$\mathrm{CV}=$ Chlorella vulgaris; $\mathrm{ISO}=$ Isochrysis $\mathrm{sp} . ; \mathrm{TP}=$ Thalassiosira pseudonana $; \mathrm{PTRI}=$ Phaeodactylum tricornutum; $\mathrm{TFL}=$ Thalassiosira fluviatilis $; \mathrm{CMU}=$ Chaetoceros muelleri $; \mathrm{NANO}=$ Nannochloropsis oculata $;$ $\mathrm{TS}=$ Tetraselmis suecica $; \mathrm{TCHU}=$ Tetraselmis chuii; ISOGA= Isochrysis galbana .

\begin{tabular}{|c|c|c|c|c|c|c|c|c|c|c|}
\hline NCFA & $\mathrm{CV}$ & ISO & $\mathrm{TP}$ & PTRI & TFL & $\mathrm{CMU}$ & NANO & TS & TCHU & ISOGA \\
\hline 08:0 & - & - & - & 0.63 & - & 0.11 & - & - & - & 0.88 \\
\hline 10:0 & - & 0.97 & & & & & & & & \\
\hline $12: 0$ & - & - & - & - & - & - & 0.31 & - & - & - \\
\hline $14: 0$ & 1.72 & 15.47 & 0.60 & 5.95 & 13.35 & 11.87 & 8.14 & 0.56 & 1.51 & 21.65 \\
\hline $14: 1$ & 1.15 & 0.92 & 13.75 & - & - & - & - & 0.13 & 0.97 & 3.98 \\
\hline $15: 0$ & - & - & 0.51 & 1.38 & & & & & & \\
\hline $16: 0$ & 21.17 & 12.61 & 2.69 & 26.96 & 3.35 & 0.96 & 33.17 & 27.32 & 23.43 & 20.84 \\
\hline 16:1 & 8.03 & 3.59 & 24.21 & - & 16.12 & - & 30.96 & 5.56 & 5.25 & 3.693 \\
\hline $17: 0$ & 1.29 & - & 27.33 & 2.73 & - & - & - & 1.02 & 0.473 & 0.99 \\
\hline 18:0 & 15.47 & 1.59 & 3.11 & 6.09 & 37.95 & 14.38 & 1.07 & 12.68 & 2.68 & 4.72 \\
\hline 18:1 & - & 18.29 & 4.51 & 4.19 & 7.07 & - & 13.53 & 25.11 & 17.55 & 21.12 \\
\hline $18: 1 \mathrm{c}$ & 13.46 & & & & & & & & & \\
\hline $18: 1 \mathrm{t}$ & 0.89 & 0.94 & 1.80 & & & & & & & \\
\hline 18:2 & 7.44 & 3.54 & 3.38 & 3.57 & 1.22 & & & & & \\
\hline $18: 2 \mathrm{t}$ & - & - & - & - & - & 0.243 & - & - & 0.20 & 0.193 \\
\hline $18: 2 \mathrm{c}$ & - & - & - & - & - & - & - & 2.23 & 6.19 & 0.873 \\
\hline $18: 3 d$ & 22.17 & 18.36 & 3.99 & 1.71 & 6.69 & 2.84 & 2.43 & 14.57 & 17.67 & 5.54 \\
\hline $18: 3$ & - & - & - & - & - & - & - & 3.88 & - & 1.20 \\
\hline 20:0 & 0.21 & 9.46 & 1.46 & - & - & - & 3.23 & - & 18.32 & 10.91 \\
\hline $20: 3$ & 0.57 & - & 3.33 & - & 7.12 & 9.69 & - & - & 1.51 & 0.87 \\
\hline 20:4 & - & - & - & - & - & - & - & 0.12 & & \\
\hline $20: 5$ & 0.43 & 3.05 & 2.15 & 13.03 & - & 0.12 & 5.36 & 5.24 & & - \\
\hline 22:0 & - & 0.16 & - & - & 0.76 & 0.157 & 0.95 & - & 3.61 & - \\
\hline $22: 1$ & - & - & 0.397 & - & - & - & - & - & 0.43 & \\
\hline $22: 2$ & 0.44 & 2.18 & & & & & & & & \\
\hline $22: 6$ & 0.86 & 0.18 & - & - & - & 2.12 & 0.973 & - & - & 3.92 \\
\hline $24: 0$ & - & 1.48 & 1.01 & 3.11 & 1.87 & 1.88 & - & - & - & 1.38 \\
\hline $24: 1$ & - & 0.67 & & & & & & & & \\
\hline Total & 97.32 & 95.10 & 93.98 & 69.34 & 98.18 & 100.14 & 100.13 & 100.00 & 100.00 & 99.80 \\
\hline
\end{tabular}

$\mathrm{NCFA}=$ Number of carbons on fatty acid chains.

According to Schneider et al. (1995), the most abundant fatty acids in the genus Nannochloropsis are $16: 0,16: 1_{n-7}$ and $20: 5_{n-3}$, a profile that was confirmed in the present study (Table 2). Tonon et al. (2002) observed large variations in the fatty acid profile, depending on the extraction method and the growth phase during which the analysis was made, although there was a predominance of the fatty acids 16:1, 16:0,18:1 and 20:5 in N. oculata, a tendency that was confirmed for this species in the present study (Table 2).

SFAs (41.91\%) were predominant in T. suecica, followed by MUFAs (30.79\%) and PUFAs (26.03\%). T. suecica contained high levels of the SFAs 16:0 (27.32\%) and 18:0 (12.68\%), followed by the MUFA
18:1 $(25.11 \%)$ and the PUFAs 18:3 $\delta(14.57 \%)$ and 20:5 (5.24\%) (Table 2). Similar results were also reported by Servel et al. (1994), although the authors found a high level of the fatty acid 18:4 ${ }_{n-3}(21.6 \%)$. SFAs were predominant in T. chuii (50.02\%), followed by PUFAs (25.58\%) and MUFAs (24.19\%); the latter two were found at similar levels in the present study (Table 1). The highest values for $T$. chuii were for the SFA 16:0 palmitic acid (23.43\%) and 20:0 eicosanoic acid (18.32\%), followed by the PUFA 18:3/ $\delta$-linolenic acid (17.67\%) and the MUFA 18:1/ oleic acid (17.55\%) (Table 2).

The highest mean fatty acid percentage in $I$. galbana was of SFAs (58.04\%), followed by MUFAs (28.80\%) and at lower levels PUFAs (12.57\%), 
as shown in Tables 1 and 2. I. galbana contained higher levels of the SFA 14:0 tetradecanoic acid $(21.65 \%)$ and $16: 0$ palmitic acid $(20.84 \%)$, followed by the MUFA 18:1 oleic acid (21.12\%), the SFA 20:0 eicosanoic acid $(10.91 \%)$ and the PUFA 18:38-linolenic acid $(5.44 \%)$, as shown in Table 2. Similar results were reported by Servel et al. (1994) for the same species, although the authors reported a high percentage of the PUFA 18:4 ${ }_{n-3}$, which was not observed in the present study. Poisson \& Ergan (2001) reported high levels of the fatty acids 18:1, 14:0, 16:0 and 22:3 for I. galbana in cultures grown in Jones or Provasoli 1/3 medium; regardless of whether the cultures were analysed at day 4, 8 or 14 , higher levels of the fatty acid 18:1 were always observed. In the present study there were similarities in the values obtained for the fatty acids 14:0, 16:0 and 18:1. High levels of the fatty acids 14:0, 16:0 and 18:1 were also previously reported for I. galbana (Qi et al., 2003).

Sánchez et al. (2000) reported high percentages of 14:0 tetradecanoic acid (27.8\%), 16:0 (20.5\%), 18:1 (10.4\%), and 22:6 ${ }_{(\mathrm{n}-3)} / \mathrm{DHA}(9.7 \%)$ in I. galbana. In the present study values of $21.65 \%, 20.84 \%$, $21.12 \%$ and $3.9 \%$ were obtained for the same fatty acids, respectively, which are very similar to the above-cited results. According to Qi et al. (2002), I. galbana is rich in PUFAs such as DHA $\left(\mathrm{C} 22: 6_{\mathrm{n}-3}, \Delta^{4,7,10,13,16,19}\right.$ ) which represents $12 \%$ of total fatty acids and is thus higher than the mean value obtained in the present study (Table 2). The above-mentioned authors also obtained a higher value for the fatty acid 18:3, a similar value for 14:0 and lower values for 16:0, 16:1 and 18:1.

The highest fatty acid concentration in C. vulgaris was that of the PUFA 18:3 (22.17\%), followed by the SFAs 16:0 (21.17\%), 18:0 (15.47\%) and 18:1 (13.46\%) (Table 2). Similarly, Mendes et al. (1995) reported the fatty acids $18: 1,16: 0$ and $18: 3$ to be the main lipid constituents, representing $41 \%, 22 \%$ and $9 \%$, respectively, and thus exhibiting higher, similar and lower levels, respectively, than those found in the present study (Table 2). The highest percentage of fatty acids in C. vulgaris was of SFAs (40.2\%), followed by PUFAs $(31.9 \%)$ and a lower MUFA content (23-5\%), as shown in Table 2.

According to Ramadhas et al. (2005), soybean oil has the following profile: $11.75 \%$ 16:0/palmitic acid, 3.15\% 18:0/stearic acid, $23.27 \%$ 18:1/oleic acid, 55.53\% 18:2/linoleic acid and 6.31\% 18:3/ linolenic acid. As shown in Table 2, the fatty acid profile of C. vulgaris is much more complex and the percentages of linoleic and oleic acids are much lower, whereas the percentages of palmitic, stearic and linolenic acids are higher.

The highest percentage of fatty acids in T. pseudonana was that of MUFAs $(44.67 \%)$, followed by SFAs $(36.72 \%)$ and PUFAs $(12.85 \%)$. Among SFAs, the highest percentage was that of 17:0/heptadecanoic or margaric acid, with $27.33 \%$ of the total fatty acid content. High levels of the MUFAs 14:1 (13.75\%) and 16:1 (24.21\%) were observed. The highest PUFA level was that of the fatty acid 18:3, although this was similar to the values obtained for 18:2 and 20:3 (Table 2). Tonon et al. (2002) observed the following profile for T. pseudonana: $7.28 \%$ 14:0, $22.64 \%$ 16:0, $26.15 \%$ $16: 1,1.94 \% 18: 0,5.30 \% 18: 1,16.66 \% 20: 5$ and $4.54 \% 22: 6$, with a predominance of the fatty acids 16:1, 16:0 and 20:5, a tendency that was maintained independently of the extraction method and the growth phase during which the analysis was made. However, this tendency was not confirmed for this species in the present study (Table 2).

There was a high percentage of non-determined fatty acids in P. tricornutum, comprising $30.66 \%$ of the total fatty acid content. However, the predominance of SFAs, mainly 16:0, followed by the PUFA 20:5 (Table 2), was observed, which is typical for Diatomaceae during cold periods. High levels of the fatty acids 20:5, 16:1, 16:0 and 14:0 were reported by Alonso et al. (2000) for P. tricornutum, and these differ from the profile obtained for the same species in the present study (Table 2).

In Isochrysis sp., the highest fatty acid content was that of the PUFA 18:3 (18.36\%), followed by the MUFA 18:1 (18.29\%) and the SFA 14:0 $(15.47 \%)$. However, the highest fatty acid percentage in Isochrysis sp. was for SFAs (41.73\%), followed by PUFAs $(27.30 \%)$, whereas the lowest content was that of MUFAs (24.41\%) (Table 1). Renaud et al. (1999) reported higher levels of the SFAs 14:0 (17.3\%) and 16:0 (12.0\%), the MUFA 18: $1_{n-7}(6.9 \%)$ and the PUFAs $18: 4_{\mathrm{n}-3}(19.0 \%), 18: 5_{\mathrm{n}-3}(10.6 \%)$ and $22: 6_{\mathrm{n}-3}(9.9 \%)$ for Isochrysis sp. Cultivating Isochrysis sp. under different temperatures, Renaud et al. (2002) reported the highest levels for SFA 14:0 (25.9\%), the MUFA $16: 1_{n-7}(11.3 \%)$ and the PUFA $18: 4_{n-3}(10.0 \%)$ at a temperature of $25^{\circ} \mathrm{C}$. In the present study, high levels of the SFAs 14:0, 16:0 and 20:0, the MUFA 18:1 and the PUFA 18:3 were observed for Isochrysis sp. (Table 2). 


\section{Conclusions}

The species $N$. oculata exhibited the highest lipid content, with low levels of polyunsaturated fatty acids and high levels of monounsaturated fatty acids, which together comprised more than $50 \%$ of the total fatty acids.

The species T. pseudonana exhibited a high lipid content, high levels of monounsaturated fatty acids and low levels of polyunsaturated fatty acids.
The species C. vulgaris and Isochrysis sp. exhibited high levels of polyunsaturated fatty acids. With regard to LC-PUFAs (long-chain polyunsaturated fatty acids), the species $P$. tricornutum, $N$. oculata and $T$. suecica exhibited the highest levels of EPA (eicosapentaenoic acid), and the species I. galbana and C. muelleri exhibited the highest levels of DHA (docosahexaenoic acid).

The freshwater species $C$. vulgaris had the highest levels of linoleic and linolenic acids, followed by the marine species $T$. chuii.

\section{Literature Cited}

Alonso, D.L.; Belardi, E-H.; Fernández-Sevilha, J.M.; RodriguezRuiz, J.; Grima, E.M.

2000. Acyl lipid composition variation related to culture age and nitrogen concentration in continuous culture of the microalga Phaeodactylum tricornutum. Phytochemistry, 54: 461-471.

Brown, M.R.; Jeffrey, S.W.; Volkman, J.K.; Dunstan, G.A. 1997. Nutritional properties of microalgae for mariculture. Aquaculture, 151: 315-331.

Castro Araújo, S.; Garcia, V.M.T.

2005. Growth and biochemical composition of the diatom Chaetoceros cf. Wighamii brightwell under different temperature, salinity and carbon dioxide levels. I. Protein, carbohydrate and lipids. Aquaculture, 246: 405-412.

Derner, R.B.; Ohse, S.; Villela, M.; Carvalho, S.M. de; Fett, R. 2006. Microalgas, produtos e aplicações. Ciência Rural, 36 (6): 1959-1967

Guillard, R.R.L.

1975. Culture of phytoplankton for feeding marine invertebrates. In: Smith, W.L. e Chanley, M.H. (Eds.) Culture of Marine Invertebrate Animals. Plenum, New York, 29-60.

Guillard, R.R.L.; Lorenzen, C.J.

1972. Yellow-green algae with chlorophyllid-c. Journal of Phycology, 8: 10-14.

López-Elías, J.A.; Voltolina, D.; Enríquez-Ocaña, F.; GallegosSimental, G.

2005. Indoor and outdoor mass production of the diatom Chaetoceros muelleri in a Mexican commercial hatchery. Aquacultural Engineering, 33: 181-191.

López-Elías, J.A.; Voltolina, D.; Enríquez-Ocaña, F.; GallegosSimental, G.

2003. Mass production of microalgae in six commercial shrimp hatcheries of the Mexican northwest. Aquacultural Engineering, 29: 155-164.

Mata, T.M.; Martins, A.A.; Cetano, N.S.

2010. Microalgae for biodiesel production and other applications: a review. Renewable and Sustainable Energy Reviews, 14: 217-232.

Medina, R.A.; Molina G.E.; Giménez, G.A.; Gonzáles, I.M.J. 1998. Downstream processing of algal polyunsaturated fatty acids. Biotechnology Advances, 16 (3): 517-580.

Mendes, R.L.; Fernandes, H.L.; Coelho, J.P.; Reis, E.C.; Cabral,

J.M.S.; Novais, J.M.; Palavra, A.F.

1995. Supercritical $\mathrm{CO}_{2}$ extraction of carotenoids and other lipids from Chlorella vulgaris. Food Chemistry, 53: 99-103.
Miao, X.; Wu, Q.

2004. High yield bio-oil production from fast pyrolysis by metabolic controlling of Chlorella protothecoides. Journal of Biotechnology, 110: 85-93.

Michael, A.; Borowitzka, M.A.

1988. Fats, oil and hydrocarbons. In: Borowitzka, M.A., Borowitzka, L.J. (eds). Microalgal Biotechnology, Cambridge University Press, USA, 257-287.

Nevejan, N.; Saez, I.; Gajardo, G.; Sorgeloos, P.

2003. Supplementation of EPA and DHA emulsions to a Dunaliella tertiolecta diet: effect on growth and lipid composition of scallop larvae, Argopecten purpuratus (Lamarck, 1819). Aquaculture, 217: 613-632.

Poisson, L.; Ergan, F.

2001. Docosahexaenoic acid ethyl esters from Isochrysis galbana. Journal of Biotechnology, 91: 75-81.

Qi, B.; Beaudoin, F.; Fraser, T.; Stobart, K.; Napier, J.A.; Lazarus, C.M

2002 Identification of a cDNA encoding a novel C18- $\Delta^{9}$ polyunsaturated fatty acid-specific elongating activity from the docosahexaenoic acid (DHA) producing microalga Isochrysis galbana. FEBS Letters, 510: 159-165.

Qi, B.; Beoxiu, F.; Fraser, T.; Bleakley, C.L.; Shaw, E.M.; Stobart, K.; Lazarus, C.M.

2003. The variant his-boxof the C18- $\Delta$ 9-PUFA-specific elongase IgASE1 from Isochrysis galbana is essential for optimum enzyme activity. FEBS Letters, 547: 137-139.

Ramadhas, A.S.; Jayaraj, S.; Muraleedharan, C.

2005. Biodiesel production from high FFA rubber seed oil. Fuel, 84: 335-340.

Rebolloso-Fuentes, M.M.; García, F.N.; Guil-Guerrero, J.L. 2001. Biomass nutrient profiles of the microalga Phaeodactylum tricornutum. Journal of Food Biochemistry, 25: 57-76.

Renaud, S.M.; Thinh, L.V.; Lambrinidis, G.; Parry, D.L.

2002. Effect of temperature on growth, chemical composition and fatty acid composition of tropical Australian microalgae grown in batch cultures. Aquaculture, 211: 195-214.

Renaud, S.M.; Thinh, L.V.; Parry, D.L.

1999. The gross chemical composition and fatty acid composition of 18 species of tropical Australian microalgae for possible use in mariculture. Aquaculture, 170: 147-159. 
Richmond, A.

2004. Handbook of Microalgal Culture: Biotechnology and Applied Phycology. Cornwall: Blackwell Publishing, 566 p. Rousch, J.M.; Bingham, S.E.; Sommerfeld, M.R.

2003. Changes in fatty acid profiles of thermo-intolerant and thermo-tolerant marine diatoms during temperature stress. Journal of Experimental Marine Biology and Ecology, 295: 145-156.

Saavedra, M.P.S.; Voltolina, D.

1994. The chemical composition of Chaetoceros sp. (Bacillariophyceae) under different light conditions. Comparative Biochemistry and Physiology, 1: 39-44.

Sánchez, S.; Martínez, M.E.; Espinola, F.

2000. Biomass production and biochemical variability of the marine microalga Isochrysis galbana en relation to culture medium. Biochemical Engineering Journal, 6: 13-18.
Schneider, J.C.; Livne, A.; Sukenik, A.; Roessler, P.G. 1995. A mutant of Nannochloropsis deficient in eicosapentaenoic acid production. Phytochemistry, 40 (3): 807-814.

Servel, M-O.; Claire, C.; Derrien, A.; Coiffard, L.; RoeckHoltzahauer, de Y.

1994. Fatty acid composition of some marine microalgae. Photochemistry, 36 (3): 691-693.

Tonon, T.; Harvey, D.; Larson, T.R.; Graham, I.A.

2002. Long chain polyunsaturated fatty acid production and partitioning to triacylglycerols in four microalgae. Phytochemistry, 61: 15-24.

Valenzuela-Espinoza, E.; Millan-Nunez, R.; Nunez-Cebrero, F. 2002. Protein, carbohydrate, lipid and chlorophyll a content in Isochrysis aff. galbana (Clone T-iso) cultured with a low cost alternative to the f/2 medium. Aquaculture Engineering, 25: 207-216. 
